\title{
The Myogenic Response of Arterial Vessels Is Increased in Fetal Pulmonary Hypertension
}

\author{
JAQUES BELIK \\ Departments of Pediatrics and Physiology, University of Manitoba, Manitoba, Canada R3E OL8
}

\begin{abstract}
The stretch-induced myogenic response (MR) of large-
capacitance pulmonary arteries were studied in normal and pul-
monary hypertensive fetuses as well as normal newborn and
adult sheep. Pulmonary hypertension in the fetus was induced by
ligation of the ductus arteriosus for $12 \mathrm{~d}$. The MR was obtained
by stretching the vessel segments in vitro from their resting
diameter (no load) to the diameter at which the muscle fibers
were at optimal length $\left(\mathrm{L}_{\mathrm{o}}\right)$, and the response was measured as a
percentage of force obtained after supramaximal electrical stim-
ulation ( $\mathrm{P}_{\mathrm{o}}$ ). In five control and four pulmonary hypertensive
fetuses, the MR was also obtained after a stretch of $140 \%$ of $\mathrm{L}_{\mathrm{o}}$.
The pulmonary hypertensive fetal arteries had a lower stress $(1.3$
\pm 0.4 versus $\left.4.0 \pm 0.5 \mathrm{mN} / \mathrm{mm}^{2} ; p<0.001\right)$ and shortening
capacity compared with the fetal control $(5.1 \pm 1.6$ versus $9.9 \pm$
$0.8 \%$ of $\left.\mathrm{L}_{\mathrm{o}} ; p<0.01\right)$. The MR was observed in $21 \%$ of the
control and $30 \%$ of the experimental fetuses, and it was of greater
magnitude in the latter $\left(14.8 \pm 1.9\right.$ of $\mathrm{P}_{\mathrm{o}}$ versus $34.3 \pm 2.5 \%$,
respectively; $p<0.01)$. When stretched to $140 \%$ of $\mathrm{L}_{\mathrm{o}}$, the $\mathrm{MR}$
\end{abstract}
was also greater in the experimental $\left(514 \pm 148 \%\right.$ of $\left.\mathrm{P}_{\mathrm{o}}\right)$ than the control fetuses $(142 \pm 68 ; p<0.05)$. Postnatally, the MR was present in $67 \%$ of the newborn and $15 \%$ of the adult pulmonary artery segments, and the response was greatest in the newborn $\left(23.1 \pm 4.2 \%\right.$ of $\left.\mathrm{P}_{\mathrm{o}}\right)$ compared with the adult $(2.3 \pm 0.8 ; p<$ $0.01)$. These data indicate that the stretch-induced MR of largecapacitance pulmonary arteries of the sheep shows significant developmental differences postnatally and is greatly enhanced in the fetus after pulmonary hypertension. We speculate that largecapacitance arteries play a role in the control of pulmonary vascular resistance in pulmonary hypertension as a result of wall stiffness changes in response to intravascular pressure-induced MR. (Pediatr Res 37: 196-201, 1995)

\section{Abbreviations}

PPHN, persistent pulmonary hypertension syndrome $\mathbf{L}_{\mathbf{o}}$, muscle optimal length

$\mathbf{P}_{\mathbf{o}}$, muscle maximum isometric force generation
It has long been shown that changes in intravascular pressure can stimulate smooth muscle to contract (1). This phenomenon, known as the myogenic response, can be reproduced in vitro by mechanical changes in vessel wall stress. The vascular wall receptor sites and the transduction pathways for muscle contraction associated with this response are not completely understood, but these are believed to be related to stretch-induced opening of cell-membrane calcium channels and resulting smooth muscle cell-membrane depolarization. This response has been documented in systemic as well as pulmonary vessels and is considered predominant at the level of small resistance arteries $(2,3)$.

We have recently observed the presence of myogenic response in large-capacitance pulmonary arteries of the guinea pig. Stretch-induced force generation of second-generation pulmonary arteries was seen in newborn and adult animals, with the magnitude of the response being greater in the former. Interestingly, in the newborn, the myogenic response in the

Received April 20, 1994; accepted August 25, 1994

Correspondence: Jaques Belik, M.D., Women's Hospital, Room WR 004, 735 Notre Dame Ave., Winnipeg, Manitoba R3E OL8, Canada.

Supported by a grant from The Medical Research Council of Canada. pulmonary artery is quite powerful, and a force $1000 \%$ greater than that after high-potassium stimulation was observed (4).

The smooth muscle cell's ability to generate force is dependent on the position of the actomyosin cross-bridges. After a stimulus, the smooth muscle develops maximum tension when its myofibrils are stretched to the point where the cross-bridges are ideally positioned to allow for maximal interaction (optimal length). Muscle elongation beyond the optimal length results in a decrease in force generation, presumably because of dissociation of cross-bridges.

Force generation in relation to the muscle fiber optimal length is problematic when stretch-induced myogenic response is evaluated. Most commonly, vessels are stretched by as much as $200 \%$ of their optimal length to induce the myogenic response $(2,5-7)$. Such a degree of stretch not only is nonphysiologic, but the muscle's cross-bridges may be irreversibly damaged.

It is apparent that, at least for pulmonary vessels, the myogenic response can be elicited after a lesser degree of wall stretch. We have recently shown that the myogenic response in the newborn and adult guinea pig can be elicited by stretching the pulmonary arterial smooth muscles from their resting length to optimal length (8). This represents a $20 \%$ increase in 
vessel diameter and maintains the muscle under its optimal length. We observed that the magnitude of contraction induced by this lesser stretch was also greater in the newborn, confirming that developmental differences do exist, even at levels of stretch likely to occur in vivo. This later evidence suggests that stretch-induced myogenic response of large-capacitance arteries may play a role in the developmental differences in vascular resistance.

The main objective of the present study was to evaluate the stretch-induced myogenic response of the fetal pulmonary arteries after pulmonary hypertension vis-a-vis its relevance to PPHN. The maintenance of a high pulmonary vascular resistance postnatally is the hallmark of this syndrome, but the etiology and pathogenesis of this condition are unknown.

Recently, an animal model to study PPHN was developed (9-13). Surgical ligation of the ductus arteriosus prenatally in fetal sheep results in hemodynamics and lung histologic changes similar to what is seen in infants with PPHN. We hypothesize that the large-capacitance pulmonary arteries in the fetal sheep model of PPHN exhibit an enhanced stretchinduced myogenic response compared with its normal control counterpart. Lastly, with a view to better interpret the above results, similar measurements were also obtained in newborn and adult sheep.

\section{METHODS}

Fetal studies. Twenty-four control and 16 pulmonary hypertensive fetal sheep were studied at $138 \mathrm{~d}$ gestation. Pulmonary hypertension was induced by surgical ligation of the ductus arteriosus as previously described (11). Briefly, ewes with twin pregnancy were subjected to a cesarean section under general anesthesia and the fetuses exteriorized. In the experimental animals, a left thoracotomy was performed and the ductus arteriosus was ligated with a silk band. The chest was closed, the fetuses were returned to the uterine cavity, and the incisions were closed in layers. The control fetuses were not instrumented.

At least $8 \mathrm{~d}$ after ductus arteriosus ligation, the fetuses were delivered by cesarean section (pentobarbital sodium anesthesia) and immediately killed (intracardiac potassium chloride injection). Second-generation intralobar pulmonary artery segments were dissected free immediately after death. Vessel rings $2 \mathrm{~mm}$ in width were mounted in a customized lever system and incubated for $60 \mathrm{~min}$ in Krebs-Henseleit solution bubbled with a $95 \%$ oxygen and $5 \%$ carbon dioxide and maintained at $37^{\circ} \mathrm{C}$.

Newborn and adult studies. To obtain normative data on the developmental changes in stretch-induced myogenic response in sheep, we studied newborn ( $<1$ wk old; $n=31$ ) and adult $(6-12$ mo of age; $n=20)$ sheep. The animals were killed with an overdose of pentobarbital sodium and potassium chloride, and the lungs were removed. Second-generation intralobar pulmonary arteries were dissected free and prepared as for the fetuses. The vessels were mounted as previously described, and similar measurements of their mechanical properties and myogenic response were obtained.
Smooth muscle mechanical properties. The following mechanical measurements were obtained as previously described (14): 1) Length tension curves - these were initially obtained to establish the $L_{0}$. 2) Stress - the maximal isometric tension was obtained by supramaximal electrical stimulation $\left(\mathrm{P}_{\mathrm{o}}\right)$. Stress was derived by normalizing force to the vessel crosssectional area. 3) Maximal shortening capacity-this was obtained by the quick release method, which consists of releasing the muscle to a quasi-zero load $(0.5 \mathrm{mN})$ under passive and active contraction. For the passive contraction, the degree of muscle shortening after passive recoil (releasing the muscle to a quasi-zero load from its resting tension without stimulation) was measured. For the measurements of active contraction, $2 \mathrm{~s}$ after the onset of electrical stimulation, the muscle was released to a quasi-zero load and allowed to shorten maximally. The maximum shortening capacity was represented by the difference between active and passive shortening and expressed as a percentage of $\mathrm{L}_{0}$. 4) Compliance-the vessel segment compliance was the ratio of change in vessel length over the load applied to stretch the vessel from its resting diameter (no load applied) to the diameter corresponding to the muscle fiber optimal length.

Myogenic response. To study the stretch-induced myogenic response under isometric conditions without elongating the muscle beyond its optimal length, the following steps were taken: 1) The muscle fiber $\mathrm{L}_{\mathrm{o}}$ was determined by the length tension curves, and the load to maintain the muscle at $\mathrm{L}_{\mathrm{o}}$ identified (resting load). 2) The load applied to the muscle was clamped to zero. 3) After $1 \mathrm{~min}$ at a load of zero, the resting load was reapplied such that the muscle was instantaneously returned to $\mathrm{L}_{\mathrm{o}}$. In pilot experiments, it was determined that this technique results in a vessel wall stretch equivalent to a $20 \%$ change in $\mathrm{L}_{0}$. The myogenic response was measured as the force developed over and above the resting tension (Fig. 1, panel A).

The myogenic response was also measured in the pulmonary hypertensive and control fetuses after a stretch greater than $\mathrm{L}_{\mathrm{o}}$. For that, the vessel segments were stretched up to $140 \%$ of their $\mathrm{L}_{\mathrm{o}}$. After the stretch maneuver, and when the force tracing was stable, complete muscle relaxation was induced by the addition of papaverine $\left(10^{-4} \mathrm{M}\right)$ and the stretch-induced myogenic response measured as the change in force brought about by papaverine relaxation (Fig. 1, panel B), as previously described by Hwa and Bevan (5). This degree of stretch was chosen to obtain consistent results without imposing excessive strain on the muscle's cross-bridges. The myogenic response of each vessel was elicited only once, and its magnitude was expressed as a percentage of the $\mathrm{P}_{\mathrm{o}}$ after supramaximal electrical stimulation.

Confirmation of pulmonary hypertension. To confirm the presence of chronic pulmonary hypertension in the fetuses, the right and left ventricular free wall weight ratio was obtained in the control and experimental animals. As previously shown (11), ligation of the ductus arteriosus in the fetus results in significant right ventricular hypertrophy, and increased rightto-left ventricular free wall weight ratio.

Statistical analysis. The unpaired $t$ test was used for all comparisons between control and experimental fetuses, as well 
A
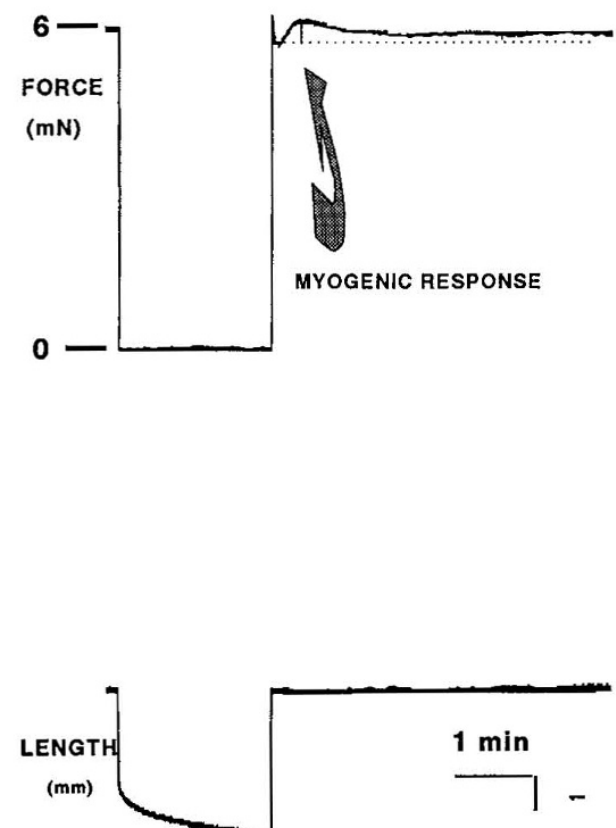

B

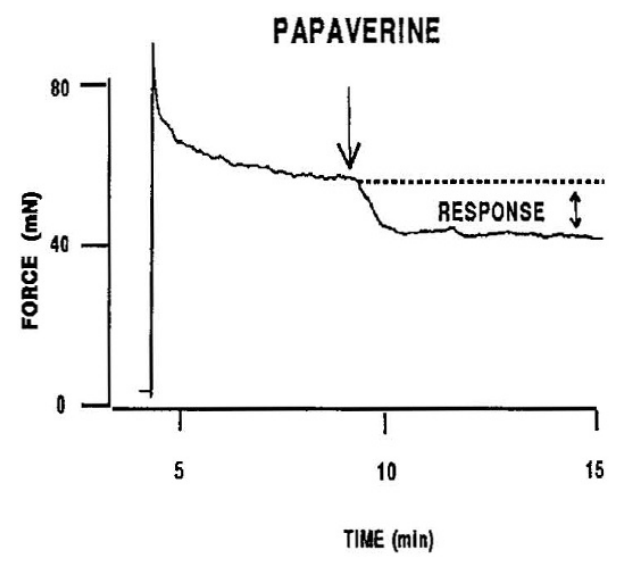

Figure 1. Representative tracing of stretch-induced myogenic response in the second-generation pulmonary artery. $A$, Wall stress induced by stretching the muscle from its resting length to the $\mathrm{L}_{\mathrm{o}}$. The myogenic response was measured as the increase in force above the resting tension (dotted line). $B$, Wall stress induced by stretching the muscle up to $140 \%$ of $\mathrm{L}_{\mathrm{o}}$. The myogenic response was measured as the decrease in force after addition of papaverine $\left(10^{-4} \mathrm{M}\right)$.

as between the newborn and adult data. Analysis of variance was used to compare the stretch-induced myogenic response in the fetus, newborn, and adult. Linear-regression analysis was used to evaluate the relationship of force and the stretchinduced myogenic response. All data are reported as mean \pm SEM. $p<0.05$ was considered significant.

\section{RESULTS}

Significant pulmonary hypertension was observed in the experimental group, as evidenced by the increased right-to-left ventricular free wall ratio of $1.38 \pm 0.11$ in the ductus arteriosus ligated versus $0.98 \pm 0.04$ in the control animals $(p<$ 0.01). Table 1 depicts the characteristics of the fetal secondgeneration intralobar pulmonary artery and their smoothmuscle isometric and isotonic mechanical properties. Pulmonary hypertension was associated with a significantly lower resting tension $(p<0.05)$, stress, and shortening capacity $(p<$ 0.01).

Table 1. Fetal vessel characteristics and smooth muscle

\begin{tabular}{lcc}
\multicolumn{3}{c}{ mechanical properties } \\
\hline & Control & $\begin{array}{c}\text { Pulmonary } \\
\text { hypertension }\end{array}$ \\
\hline Number of animals & 19 & 12 \\
Diameter (mm) & $2.9 \pm 0.1$ & $2.9 \pm 0.1$ \\
Resting tension $(\mathrm{mN})$ & $6.4 \pm 0.4$ & $4.7 \pm 0.7^{*}$ \\
Stress (mN/mm $\left.{ }^{2}\right)$ & $4.0 \pm 0.5$ & $1.3 \pm 0.4 \dagger$ \\
Shortening capacity $\left(\% \mathrm{~L}_{\mathrm{o}}\right)$ & $9.9 \pm 0.8$ & $5.1 \pm 1.6 \dagger$ \\
Compliance $(\mathrm{mm} / \mathrm{mN})$ & $0.12 \pm 0.01$ & $0.14 \pm 0.02$ \\
\hline
\end{tabular}

Values are mean \pm SEM.

${ }^{*} p<0.05$ compared with control group.

$\dagger p<0.01$ compared with control group.
Stretching the fetal pulmonary arterial segments from resting up to the $\mathrm{L}_{\mathrm{o}}$ induced an increase in force in $30 \%$ of the experimental and $20 \%$ of the control animals. The resulting stretch-induced myogenic response was 2-fold greater in the pulmonary hypertensive arterial segments compared with control values ( $p<0.01$; Fig. 2 ).

Data obtained from five control and four pulmonary hypertensive fetuses after a $140 \%$ stretch in the muscle optimal length is illustrated in Figure 3. This degree of stretch resulted in a myogenic response in $100 \%$ of the experimental and $60 \%$ of the control fetuses, with the ultimate force generated in all responders being much greater than that obtained after supramaximal electrical stimulation in both groups. Yet the average myogenic response was still 3-fold greater in the pulmonary hypertensive vessels compared with the controls $(p<0.05)$.

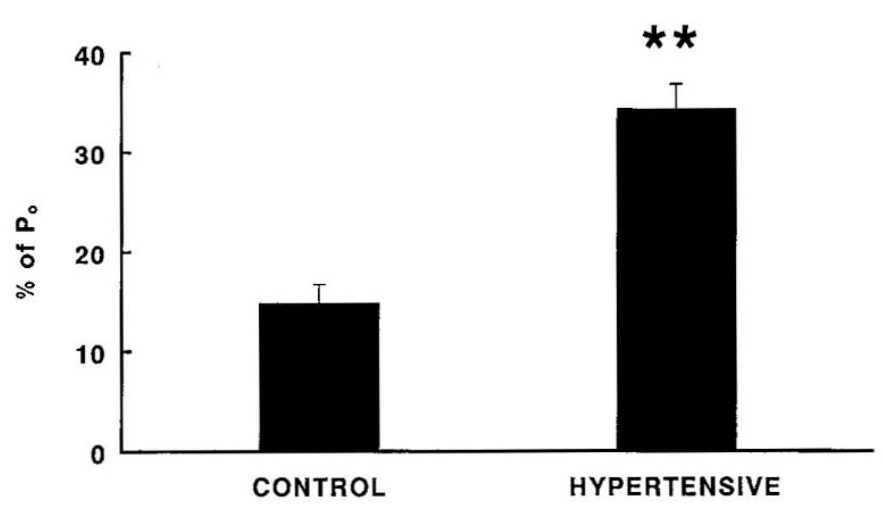

Figure 2. Stretch-induced myogenic response expressed as a percentage of the force generated after a supramaximal electrical stimulation $\left(\mathrm{P}_{\mathrm{o}}\right)$ for the fetal control $(n=19)$ and pulmonary hypertensive $(n=12)$ second-generation pulmonary arterial vessels. ${ }^{* *}, p<0.01$ compared with control. 


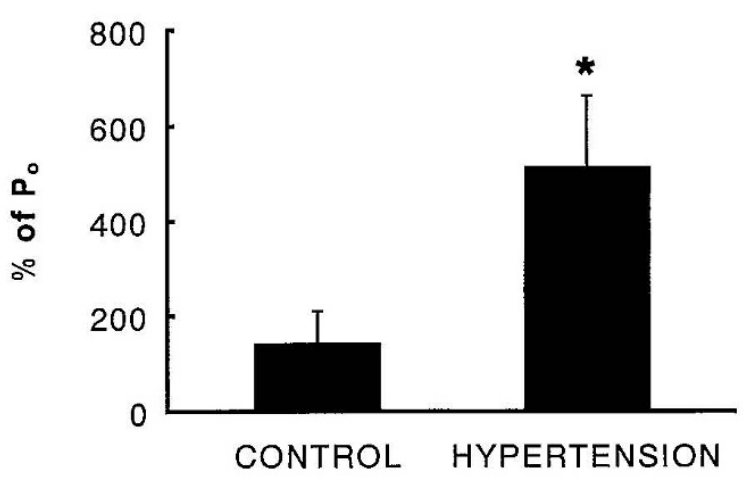

Figure 3. Myogenic response in the control $(n=5)$ and pulmonary hypertensive fetuses $(n=4)$ after a $140 \%$ of $\mathrm{L}_{\mathrm{o}}$ stretch in the vessel wall. ${ }^{*}, p<$ 0.05 .

The vessel characteristics and the smooth muscle mechanical properties of newborn and adult sheep pulmonary arteries are illustrated in Table 2. Compared with the newborn, the adult pulmonary arterial muscle stress was higher $(p<0.01)$ and the shortening capacity greater $(p<0.05)$.

A myogenic response was observed in $15 \%$ of the adult and $67 \%$ of the newborn pulmonary artery segments. As illustrated in Figure 4, significant developmental differences in the stretch induced myogenic response of large pulmonary arteries were observed. The response was the lowest in the adult and significantly different when compared with the fetus and newborn. Although there was a tendency for the newborn values to be higher than the fetal ones, the difference did not reach statistical significance.

There was no significant correlation between the magnitude of the response and stress for the normal fetal, newborn, and adult sheep, but the highest values for the myogenic responses were seen in vessels generating a low stress after supramaximal electrical stimulation (Fig. 5).

\section{DISCUSSION}

We have observed, after a sudden and significant change in wall stress, significant developmental differences in the myogenic response of sheep second-generation intralobar pulmonary arteries. The magnitude of the response is greater in the newborn and fetus compared with the adult, regardless of whether the muscle is stretched to less than or more than its optimal length. In addition, induction of pulmonary hypertension in the fetus further enhanced the magnitude of myogenic response.

Table 2. Newborn and adult sheep vessel characteristics and smooth muscle mechanical properties

\begin{tabular}{lcc}
\hline & Newborn & Adult \\
\hline Number of animals & 31 & 20 \\
Diameter $(\mathrm{mm})$ & $2.9 \pm 0.1$ & $5.0 \pm 0.3^{*}$ \\
Resting tension $(\mathrm{mN})$ & $6.8 \pm 0.4$ & $9.6 \pm 0.3^{*}$ \\
Stress $\left(\mathrm{mN} / \mathrm{mm}^{2}\right)$ & $3.8 \pm 0.9$ & $9.8 \pm 1.5^{*}$ \\
Shortening capacity $\left(\% \mathrm{~L}_{\mathrm{o}}\right)$ & $11.1 \pm 0.8$ & $15.6 \pm 1.9 \dagger$ \\
Compliance $(\mathrm{mm} / \mathrm{mN})$ & $0.12 \pm 0.01$ & $0.14 \pm 0.01$ \\
\hline
\end{tabular}

Values are mean \pm SEM.

${ }^{*} p<0.01$ compared with newborn.

$\dagger p<0.05$ compared with newborn.

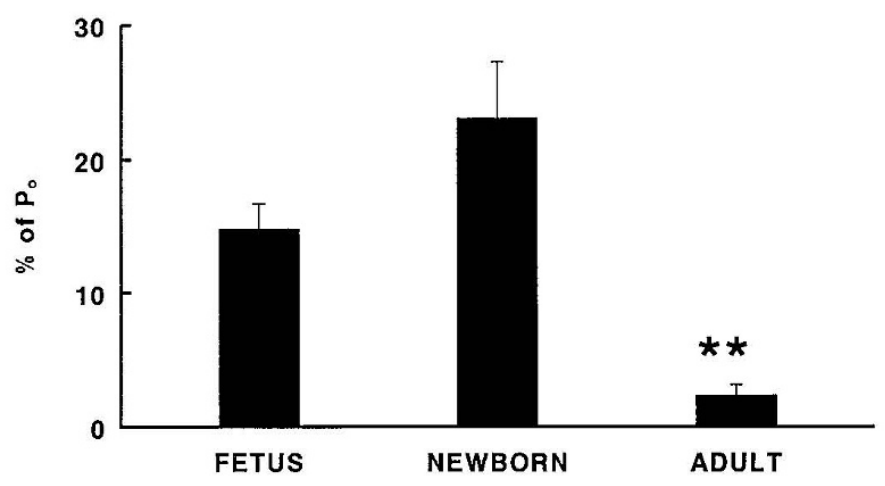

Figure 4. Stretch-induced myogenic response (from resting to optimal length) expressed as a percentage of the supramaximal electrical stimulation force for the normal fetal, newborn, and adult sheep. ${ }^{* *}, p<0.01$ compared with the fetal and newborn groups.

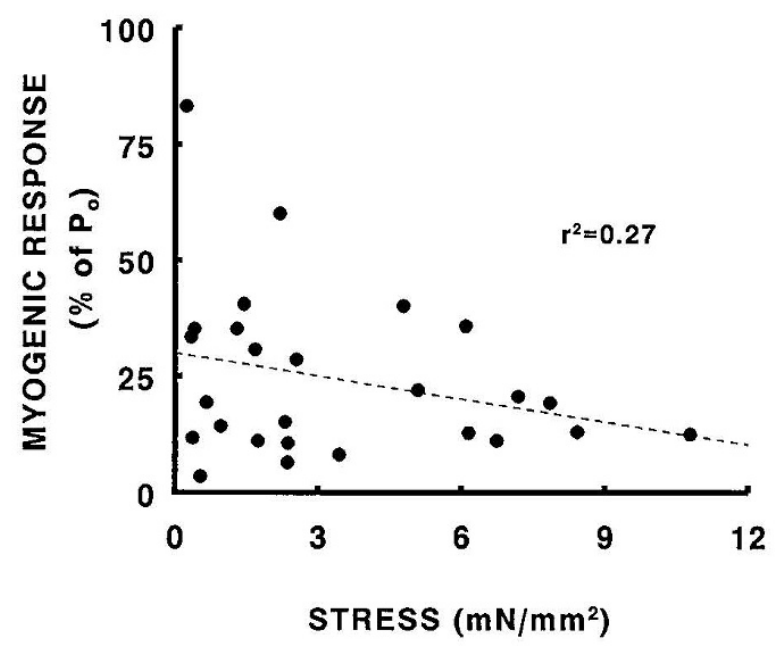

Figure 5. Stretch-induced myogenic response expressed as a percentage of force generated by a supramaximal electrical stimulation in normal fetal, newborn, and adult vessels plotted against their respective stress values. The regression line is depicted by the broken line $\left(R^{2}=0.27, p=\mathrm{NS}\right)$.

To evaluate the stretch-induced myogenic response under conditions comparable to the conditions in vivo, we initially stretched the vessel segments by only $20 \%$ of their resting diameter. Other investigators have used much greater degrees of in vitro wall stress, stretching the vessels by as much as $200 \%$ of their resting diameter (5-7). In eliciting this lesser degree of wall stretch, the percentage of vessels responding with force development (myogenic response) varied by as little as $15 \%$ in the adults and $67 \%$ in the newborn. In the fetuses, the myogenic response was obtained in $100 \%$ of the pulmonary hypertensive vessels after a stretch of up to $140 \%$ of the $\mathrm{L}_{\mathrm{o}}$, and the force generation in the responder controls and experimental vessels was much greater than that obtained with electrical stimulation. Presently, the factors accounting for lack of response in certain vessels are unclear. Nevertheless, in the fetus, a stretch-induced force greater than that after supramaximal electrical stimulation suggests that changes in wall stress are a very powerful stimulus for muscle contraction.

The myogenic response has been studied in systemic and pulmonary vessels (15). Although it is observed in both large and small resistance arteries, it is believed to be more prominent in the latter (15). Limited data are available on the 
myogenic response of pulmonary arteries. In the adult cat, stretch-induced force development in vitro was only seen in vessels with a diameter $<1000 \mu \mathrm{m}$ (16). In the newborn and adult guinea pig, we have observed a significant myogenic response in large-capacitance arteries $(4,8)$. As observed for the sheep, the newborn guinea pigs' response is much greater than the adult's, and the magnitude of the force is proportional to the degree of stretch. In the newborn, after a stretch of $200 \%$ of the vessel diameter, the muscle generates a force 10 times greater than that obtained with high potassium stimulation in the newborn (4). In contrast, the adult guinea pig's large pulmonary arteries develop a maximal force only 3 times greater than that observed with high potassium stimulation after a $160 \%$ increase in vessel diameter. Thus, on the basis of data obtained from these two animal species, it is evident that not only is the myogenic response present in large-capacitance arteries, but it can amount to significant force development, even compared with other potent smooth muscle contraction stimuli. Notwithstanding, it is important to emphasize that, although each vascular muscle was studied at its optimal length and thus under conditions similar to those in vivo, the present in vitro data cannot be readily extrapolated. In vivo changes in vessel diameter with smooth muscle activation might result in myogenic responses different from those observed in vitro; thus, additional whole-organ studies are required to validate the present data.

The factors accounting for the maintenance of a higher pulmonary vascular resistance in PPHN of the newborn are unknown (17). Most studies using the prenatally ductus arteriosus-ligated fetal sheep model of pulmonary hypertension have concentrated on in vivo hemodynamic measurements ( 9 , $10,12,13)$. Abnormalities in nitric oxide released by the endothelium (18) or an increased synthesis of vasoactive peptide such as endothelin-1 (19) are believed to act on small resistance arteries, resulting in the maintenance of a higher pulmonary vascular resistance postnatally in this model $(20$, 21). Histologically, the lung changes associated with pulmonary hypertension are limited to extension of muscle to nonmuscular peripheral arteries, suggesting that solely the small resistance arteries are responsible for the hemodynamic changes in the fetal sheep animal model and in human disease.

In contrast, we have recently reported significant abnormalities in the smooth muscle of large-capacitance pulmonary arteries of the ductus arteriosus-ligated fetal sheep (11). The pulmonary vascular smooth muscle generates less force and has a smaller shortening capacity but has a much longer relaxation time. The present data demonstrate that the smooth muscle of these arteries exhibits a stretch-induced myogenic response of greater magnitude than controls. These data prompted us to further speculate on the role of the largecapacitance pulmonary arteries in the control of pulmonary vascular resistance.

It is well known that the large-capacitance arteries play an important role in the maintenance of adequate circulation by "storing" energy during systole and returning it as elastic recoil during diastole, thereby maintaining blood flow (Windkessel effect). In addition, the large elastic arteries "buffer" the pressure transmission of the pulse wave to the smaller resistance ones (22). Inasmuch as the degree of vasoconstriction of smaller resistance arteries is directly dependent on the intravascular pressure (myogenic response), this ability of the larger-capacitance vessels to reduce pressure transmission to the smaller arteries likely makes them an integral participant in the control of pulmonary vascular resistance. In this respect, we speculate that wall stretch of large-capacitance arteries caused by the pulse wave results in stretch-induced myogenic response of their musculature and stiffening of their wall. Other investigators have made similar speculations in the past (23), suggesting that the elastic properties of these vessels will be counteracted by muscle contraction and that the pressure transmitted downstream is directly proportional to the degree of stiffness of these vessels.

Our previous observation of a lower stress and shortening capacity of pulmonary vascular smooth muscle in the pulmonary hypertensive fetus (11) suggests that the maintenance of a higher pulmonary vascular resistance postnatally in these animals might be the result of abnormalities in vasodilation and not of a greater potential for vasoconstriction. In this respect, the longer relaxation time and increased myogenic response of the smooth muscle in pulmonary hypertension could account for the maintenance of increased wall stiffness in largecapacitance vessels. Induction of the myogenic response with each pulse wave associated with a failure of the muscle to completely relax with each stimulation due to its increased relaxation time would result in greater vessel wall stiffness in pulmonary hypertension. Lastly, the vessel wall compliance in the control and pulmonary hypertensive animals were similar, suggesting that any differences in wall stiffness are secondary to changes in the degree of muscle contraction and not caused by altered extracellular matrix content, a common finding in neonatal and adult pulmonary hypertension $(16,24)$. This is in keeping with our previous observation of unaltered collagen and elastin content in large pulmonary arteries of the ductus arteriosus-ligated fetal sheep (25).

In conclusion, we observed a significant enhancement of the stretch-induced myogenic response after pulmonary hypertension in the fetal sheep. In addition, this response is clearly present in large-capacitance pulmonary arteries and of greater magnitude in the normal newborn and fetal sheep. Studies addressing the role of the stretch-induced myogenic response of large-capacitance arteries in the control of pulmonary vascular resistance in normal and pulmonary hypertensive fetuses are presently under way to further address this issue.

\section{REFERENCES}

1. Bayliss WM 1902 On the local reactions of the arterial wall to changes of internal pressure. J Physiol 28:220-231

2. Bevan JA, Laher I 1991 Pressure and flow-dependent vascular tone. FASEB J 5:2267-2273

3. Johnson PC 1980 The myogenic response. In: Bohr DF, Somlyo AP, Sparks HV (eds) Handbook of Physiology: The Cardiovascular System, Vascular Smooth Muscle. American Physiological Society, Bethesda, MD, pp 409-442

4. Belik J 1994 Myogenic response in large pulmonary arteries and its ontogenesis. Pediatr Res 36:34-40

5. Hwa JJ, Bevan JA 1986 Stretch dependent (myogenic) tone in rabbit ear resistance arteries. Am J Physiol 250:H87-H95

6. Jackson PA, Duling BR 1989 Myogenic response and wall mechanics of arterioles. Am J Physiol 257 26:H1147-H1155

7. Kulik TJ, Evans JN, Gamble WJ 1988 Stretch-induced contraction in pulmonary arteries. Am J Physiol 255:H1391-H1398 
8. Belik J 1995 Large pulmonary arteries and the control of pulmonary vascular resistance in the newborn. Can J Physiol Pharmacol (in press)

9. Abman SH, Accurso FJ 1989 Acute effects of partial compression of ductus arteriosus on fetal pulmonary circulation. Am J Physiol 257:H626-H634

10. Abman SH, Shanley PF, Accurso FJ 1989 Failure of postnatal adaptation of the pulmonary circulation after chronic intrauterine pulmonary hypertension in fetal lambs. J Clin Invest 83:1849-1858

11. Belik J, Halayko AJ, Rao K, Stephens NL 1993 Fetal ductus arteriosus ligation. Circ Res 72:588-596

12. Morin III FC 1989 Ligating the ductus arteriosus before birth causes persistent pulmonary hypertension in the newborn lamb. Pediatr Res 25:245-250

13. Morin III FC, Egan EA 1989 The effect of closing the ductus arteriosus on the pulmonary circulation of the fetal sheep. J Dev Physiol 11:283-287

14. Belik J, Stephens NL 1993 Developmental differences in vascular smooth muscle mechanics in pulmonary and systemic circulations. J Appl Physiol 74:682-687

15. Meininger GA, Davis MJ 1992 Cellular mechanisms involved in the vascular myogenic response. Am J Physiol 263:H647-H659

16. Mecham RP, Whitehouse LA, Wrenn DS, Parks WC, Griffin GL, Senior RM, Crouch EC, Stenmark KR, Voelkel NF 1987 Smooth muscle-mediated connective tissue remodeling in pulmonary hypertension. Science 237:423-426

17. Walsh-Sukys MC 1993 Persistent pulmonary hypertension of the newborn. Clin Perinatol 20:127-143
18. Steinhorn RH, Russell JA, Morin FC 1994 Pulmonary arteries from newborn lambs with persistent pulmonary hypertension have decreased sensitivity to nitric oxide. Pediatr Res 35:354A(abstr)

19. Cassin S, Kristova V, Davis T, Kadowitz P, Gause G 1991 Tone-dependent responses to endothelin in the isolated perfused fetal sheep pulmonary circulation in situ. J Appl Physiol 70:1228-1234

20. Zayek M, Wild L, Roberts JD, Morin III FC 1993 Inhaled nitric oxide increases survival in newborn lambs with persistent pulmonary hypertension. Pediatr Res 33:244(abstr)

21. Zayek M, Cleveland D, Morin III FC 1993 Inhaled nitric oxide selectively dilates the pulmonary circulation in newborn lambs with persistent pulmonary hypertension. Pediatr Res 33:350(abstr)

22. Best CH, Taylor NB 1991 Physiological Basis of Medical Practice. Williams \& Wilkins, Baltimore, pp 118-119

23. Mellander S, Arvidson S 1974 Possible "dynamic" component in the myogenic vascular response related to pulse pressure distension. Acta Physiol Scand 90:283285

24. Stenmark K, Fasules J, Hyde D, Voelkel N, Henson J, Tucker A, Wilson H, Reeves J 1987 Severe pulmonary hypertension and arterial adventitial changes in newborn calves at 4,300 m. J Appl Physiol 62:821-830

25. Belik J, Keeley FW, Baldwin F, Rabinovitch M 1994 Pulmonary hypertension and vascular remodelling in the fetal sheep. Am J Physiol 35:H2303-H2309 